\title{
Clinical Profile of Patients Based on Receptor Status in Carcinoma Breast
}

\author{
Pavan Chandhar Dudde ${ }^{1}$, Poorna Chandra Thejeswi ${ }^{2}$, Shivananda H Prabhu ${ }^{3}$, \\ Prathibha B Naik ${ }^{4}$, Ashwin Kumar $C^{5}$ \\ ${ }^{I}$ (Senior Resident, Department Of General Surgery, Kasturba Medical College, Manipal university, India) \\ ${ }^{2}$ (Associate Professor, Department Of General Surgery, Kasturba Medical College, Manipal university, India) \\ ${ }_{3}^{3}$ (Professor, Department Of General Surgery, Kasturba Medical College, Manipal university, India) \\ ${ }_{5}^{4}$ (Senior Resident, Department Of General Surgery, Kasturba Medical College, Manipal university, India) \\ ${ }^{5}$ (Senior Resident, Department Of General Surgery, Kasturba Medical College, Manipal university, India)
}

\begin{abstract}
Aims \& Objectives: The aim of this study is to determine the factors affecting receptor status in carcinoma breast.

The objectives include to determine factors to be studied, analyze the clinical and histological factors in relation to receptor status \& determine the relation of factors to receptor status

Materials and Methods: A prospective time bound study was planned from Nov 2013 to August 2015 with patients getting admitted in Govt Wenlock Hospital, KMC Attavar and KMC-Ambedkar Circle Hospitals in Mangalore. The factors taken into consideration were age, family history, tumour size, lymph node involvement and grading. A 'p'value of $<0.005$ is taken as significant.

Results: In a total study of 93 cases, it was noted that patients with tumour size of 2-5cm has a significant correlation with Estrogen receptor (ER) i.e of the 52 patients with tumour size of 2-5cm, 43 patients has ER positive status. $\boldsymbol{p}$ value ( $\mathbf{0 . 0 4 6}$ ). Out of 54 patients with positive lymph nodal status, 42 patients has HER2 negative status (77.8\%). p value (0.043).

Conclusion: The results of my study implies that lymph node involvement determines the HER2 receptor status and also the tumour size determines the Estrogen receptor (ER) status. However our study could not determine any significant correlation between age and receptor status may be because of sample size being small.
\end{abstract}

Keywords: Estrogen Receptor, Human Epidermal Growth factor Receptor 2, Immuno Histo Chemistry, Progesterone Receptor.

\section{Introduction}

Breast carcinoma is a heterogeneous disease, with substantial genotypic and phenotypic diversity. In the last 10- 20 years, there have been remarkable advances in the overall outlook of carcinoma breast which lead to early detection of cancer and the development of more effective treatments that resulted in reducing the deaths due to breast carcinoma and improving the outcomes for people suffering from the disease. Recent attention has been directed singularly at molecular classifications of breast cancer. While molecular and genetic testing is very elegant, prognostic and predictive, it is expensive and not yet widely available. Also, despite the prognostic information provided by the molecular test, current reports of assay results impart little specific guidance of response to targeted and proven therapy; for example, endocrine and trastuzumab therapy for tumors expressing estrogen receptor/progesterone receptor (ER/PR) or human epidermal growth factor receptor 2 (Her2) proteins, respectively. The ImmunoHistoChemistry (IHC) classification provides both therapeutic and prognostic information.

Breast cancer patients with tumors that are estrogen receptor (ER)-positive and/or progesterone receptor (PR)-positive have lower risks of mortality after their diagnosis compared to women with ER- and/or PR-negative disease. Clinical trials have also shown that the survival advantage for women with hormone receptor-positive tumors is enhanced by treatment with adjuvant hormonal and/or chemotherapeutic regimens. However, few studies have evaluated variations in the risks of breast cancer-specific mortality across ER/PR status by either demographic or clinical characteristics.

2.1 Study Design: Prospective Study.

\section{Materials And Methods}

2.2 Study Setting: Govt Wenlock Hospital Mangalore, KMC Hospital Attavar, KMC Hospital, Ambedkar Circle. Study was conducted by the Dept Of General Surgery in Surgical units in above hospitals coming under KMC Mangalore. 
2.3 Inclusion criteria: All female patients who are primarily diagnosed to have carcinoma breast.

2.4 Exclusion Criteria: Female patients less than 18 years of age \& medically unstable patients.

2.5 Study Population: All female patients with carcinoma breast in above hospitals.

2.6 Sample Size: Time Bound Prospective Study (93).

2.7 Study Duration: NOV 2013 to August 2015 .

A prospective time bound study was planned from Nov 2013 to August 2015 with patients getting admitted in Govt Wenlock Hospital, KMC Attavar and KMC-Ambedkar Circle Hospitals in Mangalore diagnosed as carcinoma breast. The entire groups of patients getting admitted with carcinoma breast during the above period were observed and data was collected. The patients were observed with history, clinical examination and laboratory investigations and the obtained data were recorded in preset pro forma sheet during the hospital stay. The same data were analyzed periodically and at the end of study period and statistical analysis was carried out to assess the correlation between clinical profile and receptor status.

Statistical analysis of the data obtained was done using SPSS version 17.0. Chi-square test, Fischer's exact test and logistic regression analysis were used for statistical analysis of the data. A 'p' value of $<0.005$ is taken as significant. The clinical and histological factors taken into consideration were age, family history, tumour size, lymph node involvement and grading.

Total patients included in study - Sample Size -93 .

\section{Results}

\subsection{Estrogen Receptor (ER)}

Out of 93 patients, 68 patients were found to be Estrogen Receptor ER positive (73.1\%). 25 patients were found to be Estrogen Receptor ER negative (26.9\%).

\subsection{Progesterone Receptor (PR)}

63 patients out of 93 were found to be Progesterone Receptor PR positive (67.7\%). 30 patients were found to be Progesterone Receptor PR negative (32.3\%).

\subsection{HER2neu Receptor (HER2)}

28 patients out of 93 were found to be HER2 positive (30.1\%). 65 patients were found to be HER2 negative $(69.9 \%)$.

\subsection{Receptor-Triple Positive \& Triple Negative}

14 patients out of 93 were found to be triple positive $(15.1 \%)$.

13 patients out of 93 were found to be triple negative (14.0\%)

\subsection{ER - PR}

Out of 68 ER positive patients, 52 patients were found to be PR positive (76.5\%) and 16 patients were found to be PR negative (235\%). In 25 ER negative patients, 11 patients were found to be PR positive (44\%) and 14 pa tients were found to be PR negative (56\%).

\subsection{ER - HER2}

Out of 68 ER positive patients, 18 patients were found to be HER2 positive (26.5\%) and 50 patients were found to be HER2 negative (73.5\%). In 25 ER negative patients, 10 patients were found to be HER2 positive (40\%) and 15 patients were found to be HER2 negative (60\%).

\subsection{HER2 - PR}

Out of 28 HER2 positive patients, 23 patients were found to be PR positive (82.1\%) and 5 patients were found to be PR negative (17.9\%). In 65 HER2 negative patients, 40 patients were found to be PR positive $(61.5 \%)$ and 25 patients were found to be PR negative (38.5\%).

3.8. Age

Of the 93 patients studied during the study period. 10 patients were under the age group of 35 years and below $(10.8 \%) .48$ patients were under the age group of $36-50$ years $(51.6 \%)$. 28 patients were under the age group of $51-70$ years (30.1\%). 7 patients were under the age group of above 70 years $(7.5 \%)$.

\subsection{Age - ER}

Under the age group of 35 years and below, 5 patients were ER positive (50\%) and 5 patients were ER negative (50\%). Under the age group of $36-50$ years, 36 patients were ER positive $(75 \%)$ and 12 patients were ER negative (25\%). Under the age group of $51-70$ years, 20 patients were ER positive (71.4\%) and 8 patients were ER negative $(28.6 \%)$. Under the age group of above 70 years, 7 patients were found to be ER positive $(100 \%)$ and no patients reported to be ER negative (0\%).

\subsection{Age - PR}

Under the age group of 35 years and below, 8 patients were PR positive (80\%) and 2 patients were PR negative (20\%). Under the age group of $36-50$ years, 31 patients were PR positive (64.6\%) and 17 patients were PR negative (35.4). Under the age group of $51-70$ years, 17 patients were PR positive (60.7\%) and 11 patients were PR negative (39.3\%). Under the age group of above 70 years, 7 patients were found to be PR positive (100\%) and no patients reported to be PR negative ( $0 \%)$. 


\subsection{Age-HER2}

Under the age group of 35 years and below, 5 patients were HER2 positive (50\%) and 5 patients were HER2 negative (50\%). Under the age group of $36-50$ years, 14 patients were HER2 positive (29.2\%) and 34 patients were HER2 negative (70.8\%). Under the age group of $51-70$ years, 8 patients were HER2 positive (28.6\%) and 20 patients were HER2 negative $(71.4 \%)$. Under the age group of above 70 years, 1 patient was found to be HER2 positive (14.3\%) and 6 patients were reported to be HER2 negative (85.7\%) .

\subsection{Family History}

Of the 93 patients studied, only 2 patients $(2.2 \%)$ were found to have positive family history.

\subsection{Family History - ER}

Of the 2 patients with positive family history, 1 patient each was reported to be ER positive (50\%) and ER negative $(50 \%)$.

\subsection{Family History - PR}

Of the 2 patients with positive family history, both the 2 patients were reported to be PR negative (100\%).

\subsection{Family History - HER2}

Of the 2 patients with positive family history, both the 2 patients were reported to be HER2 negative (100\%).

\subsection{Tumour Size}

Of the 93 patients, 17 patients were found to be under the group of $<2 \mathrm{~cm}(18.3 \%) .52$ patients were found to be under the group of $2-5 \mathrm{~cm}(55.9 \%) .24$ patients were under the group of $>5 \mathrm{~cm}(25.8 \%)$.

\subsection{Tumour Size -ER}

Under the group of $<2 \mathrm{~cm}, 11$ patients were found to be ER positive (64.7\%) and 6 patients were found to be ER negative (35.3\%). In the group of $2-5 \mathrm{~cm}, 43$ patients were found to be ER positive (82.7\%) and 9 patients were found to be ER negative (17.\%). In the group of $>5 \mathrm{~cm}, 14$ patients were found to be ER positive (58.3\%) and 10 patients were found to be ER negative (41.73\%).

Table 1. Tumour Size \& ER

\begin{tabular}{|c|c|c|c|c|}
\hline & & & & \\
\hline & & NEGATIVE & POSITIVE & Total \\
\hline$\overline{T U M O U R}$ & $<2 \mathrm{CM}$ & 6 & 11 & 17 \\
\hline SIZE & & $35.3 \%$ & $64.7 \%$ & $100.0 \%$ \\
\hline & & $24.0 \%$ & $16.2 \%$ & $18.3 \%$ \\
\hline & $>5 \mathrm{CM}$ & 10 & 14 & 24 \\
\hline & & $41.7 \%$ & $58.3 \%$ & $100.0 \%$ \\
\hline & & $40.0 \%$ & $20.6 \%$ & $25.8 \%$ \\
\hline & $2-5 \mathrm{CM}$ & 9 & 43 & 52 \\
\hline & & $17.3 \%$ & $82.7 \%$ & $100.0 \%$ \\
\hline & & $36.0 \%$ & $63.2 \%$ & $55.9 \%$ \\
\hline Total & & 25 & 68 & 93 \\
\hline & & $26.9 \%$ & $73.1 \%$ & $100.0 \%$ \\
\hline & & $100.0 \%$ & $100.0 \%$ & $100.0 \%$ \\
\hline
\end{tabular}

$p$ value is significant ( 0.046 )

\subsection{Tumour Size -PR}

Under the group of $<2 \mathrm{~cm}, 10$ patients were found to be PR positive $(58.8 \%)$ and 7 patients were found to be PR negative (41.2\%). In the group of $2-5 \mathrm{~cm}, 36$ patients were found to be PR positive (69.2\%) and 16 patients were found to be PR negative (30.8\%). In the group of $>5 \mathrm{~cm}, 17$ patients were found to be PR positive (70.8\%) and 7 patients were found to be PR negative (29.2\%).

\subsection{Tumour Size -HER2}

Under the group of $<2 \mathrm{~cm}, 3$ patients were found to be HER2 positive (17.6\%) and 14 patients were found to be HER2 negative ( $82.4 \%)$. In the group of $2-5 \mathrm{~cm}, 14$ patients were found to be HER2 positive (26.9\%) and 38 patients were found to be HER2 negative (73.1\%). In the group of $>5 \mathrm{~cm}, 11$ patients were found to be HER2 positive (45.8\%) and 13 patients were found to be HER2 negative (54.2\%).

3.20 Lymph Nodes - Involvement

54 out of 93 patients were found to have positive lymph node status (58.1\%). 39 patients did not have lymph node involvement (41.9\%). 


\subsection{Lymph Node Involvement - ER}

In the group with positive lymph nodal involvement, 42 patients (77.8\%) were found to be ER positive and 12 patients were found to be ER negative (22.2\%). In the group with negative lymph nodal involvement, 26 patients were found to be ER positive (66.7\%) and 13 patients were found to be ER negative (33.3\%).

\subsection{Lymph Node Involvement - PR}

In the group with positive lymph nodal involvement, 35 patients were found to be PR positive (64.8\%) and 19 patients were found to be PR negative (35.2\%). In the group with negative lymph nodal involvement, 28 patients were found to be PR positive (71.8\%) and 11 patients were found to be PR negative (28.2\%).

\subsection{Lymph Node Involvement - HER2}

In the group with positive lymph nodal involvement, 12 patients were found to be HER2 positive (22.2\%) and 42 patients were found to be HER2 negative (77.8\%). In the group with negative lymph nodal involvement, 16 patients were found to be HER2 positive (41\%) and 23 patients were found to be HER2 negative (59\%).

Table 23. Lymph Nodes \& HER2

\begin{tabular}{|ll|r|r|r|}
\hline & & \multicolumn{2}{|c|}{ HER2 } & \\
\cline { 3 - 5 } & & NEGATIVE & POSITIVE & \multicolumn{1}{c|}{ Total } \\
\hline LYMPH NODES & NO & 23 & 16 & 39 \\
- INVOVEMENT & & $59.0 \%$ & $41.0 \%$ & $100.0 \%$ \\
& & $35.4 \%$ & $57.1 \%$ & $41.9 \%$ \\
\cline { 2 - 5 } & YES & 42 & 12 & 54 \\
& & $77.8 \%$ & $22.2 \%$ & $100.0 \%$ \\
& & $64.6 \%$ & $42.9 \%$ & $58.1 \%$ \\
\hline Total & 65 & 28 & 93 \\
& & $69.9 \%$ & $30.1 \%$ & $100.0 \%$ \\
& & $100.0 \%$ & $100.0 \%$ & $100.0 \%$ \\
\hline
\end{tabular}

p value significant $(0.043)$

\subsection{Grading}

11 out of 93 patients were found to be under grade I (11.8\%). 47 patients were under grade II (50.5\%). 35 patients were under grade III (37.6\%).

\subsection{Grading - ER}

In the group of grade I, 9 patients were found to be ER positive (81.8\%)and 2 patients were found to be ER negative (18.2\%). In grade II, 34 patients were found to be ER positive (72.3\%)and 13 patients were found to be ER negative (27.7\%). In the group of grade III, 25 patients were found to be ER positive (71.4\%) and 10 patients were found to be ER negative (28.6\%).

\subsection{Grading - PR}

In the group of grade I, 6 patients were found to be PR positive (54.5\%) and 5 patients were found to be PR negative (45.5\%). In grade II, 30 patients were found to be PR positive (63.8\%) and 17 patients were found to be PR negative (36.2\%). In the group of grade III, 27 patients were found to be PR positive $(77.1 \%)$ and 8 patients were found to be PR negative (22.9\%).

\subsection{Grading - HER2}

In the group of grade I, 4 patients were found to be HER2 positive (36.4\%) and 7 patients were found to be HER2 negative (63.6\%). In grade II, 11 patients were found to be HER2 positive (23.4\%)and 36 patients were found to be HER2 negative (76.6\%). In the group of grade III, 13 patients were found to be HER2 positive (37.1\%) and 22 patients were found to be HER2 negative (62.9\%).

\subsection{Age}

\section{Discussion}

With age, the risk of breast cancer increases. It is estimated that $5-7 \%$ of breast carcinomas are presented in young women ( $<35$ years) in united states, ${ }^{14}$ while in our study it is about $10.8 \%$. Age range among Indian breast cancer patients is found to be lower when compared to the Western countries with an average difference of one decade. This is likely to be due to the different age distribution of the Indian population, where only $7 \%$ of the population is above the age of 60 years. In the present study $51.6 \%$ of women were in the age group of 36-50 years, in contrast a study by Pakseresht et $\mathrm{al}^{9}$ had lower age range from 31-40 years $(34.5 \%)$, whereas Ambroise et $\mathrm{al}^{6}(46.4 \%)$ Suvarchala et $\mathrm{al}^{8}(45.31 \%)$, and Rhodes et $\mathrm{al}^{10}(36.42 \%)$ had 
higher age range between 51-60years.In Europe and America, the reports of many studies suggests that breast carcinoma in young women indicates poor survival. Those studies also suggests that younger women tend to have more negative receptors than that of older women. These reports remains controversial, as Chia et al from Singapore suggested that younger women had a better survival than old women. However our study could not determine any significant correlation between age and receptor status may be because of sample size being small.

\subsection{Family History}

Of the 93 patients studied, only 2 patients $(2.2 \%)$ were found to have positive family history. 91 patients $(97.8 \%)$ did not have any significant family history.

\subsection{Tumour Size}

From our study, it was noted that patients with tumour size of $2-5 \mathrm{~cm}$ has a significant correlation with Estrogen receptor (ER) i.e of the 52 patients with tumour size of $2-5 \mathrm{~cm}, 43$ patients has ER positive status. p value is significant ( 0.046 )

In our study, we found that patients with tumour size of $\langle 2 \mathrm{~cm}$ and $2-5 \mathrm{cms}$ were more likely to be $\mathrm{ER}+/ \mathrm{PR}+$ as compared to patients with tumour size of $>5 \mathrm{~cm}$. These results were in concordance with study by Dunnwald LK. et $\mathrm{al}^{4}$ with sample size of 155,175 and most other studies.

\subsection{Lymph Node Involvement}

In our study, we observed that there is a significant correlation between HER2 receptor and lymph node involvement. Out of 54 patients with positive lymph nodal status, 42 patients has HER2 negative status $(77.8 \%)$. p value significant $(\mathbf{0 . 0 4 3})$.

\subsection{Grading}

In the present study majority of breast tumors were grade II (50\%) followed by grade III (38\%) and grade I $(12 \%)$ which is in concordance with other studies except for one study by Ghosh et al ${ }^{5}$ having more of grade III (75\%).From our study, we also observed that Estrogen receptor (ER) has a significant correlation with Progesterone receptor (PR) i.e of the 68 ER positive patients, 52 patients has PR positive status (76.5\%). p value highly significant ( $\mathbf{0 . 0 0 4}$ ) and also HER2 receptor has a significant correlation with Progesterone receptor (PR) i.e out of 28 HER2 positive patients, 23 patients has PR positive status $(82.1 \%)$. p value significant ( 0.041 ).

\section{Conclusion}

In conclusion, our study clearly demonstrates that there is a significant correlation between clinical/ histological factors and receptor status and these factors determine the prognosis in carcinoma breast patients. The results of my study implies that lymph node involvement determines the HER2 receptor status and also the tumour size determines the Estrogen receptor (ER) status. However our study could not determine any significant correlation between age and receptor status may be because of sample size being small. Hence, more studies should focus on correlation between age and receptor status. American Society of Clinical Oncology/College of American Pathologists Guideline Recommendations for IHC testing of Estrogen and Progesterone Receptors in Breast Cancer are strongly advocated for standardization of receptor evaluation and for clinical management of breast cancer patients to provide best therapeutic options.

This study illustrates the changing trends of IHC profile of breast cancer and will serve as one of the baseline studies for future work and breast cancer management strategies.

\section{References}

[1]. American Cancer Society Cancer Facts and Figures, 2004. 2004;3:10.

[2]. Albain KS, Allred DC, Clark GM. Breast cancer outcome and predictor of outcome: are there age differentials? J Natl Cancer Inst Monogr. 1994;16:35-42.

[3]. Kollias J, Elston CW, Ellis IO, Robertson JF, Blamey RW. Early-onset breast cancer-histopathological and prognostic considerations. Br J Cancer. 1997;75:1318-23.

[4]. Dunnwald LK, Rossing MA, Li CI (2007). Hormone receptor status, tumor characteristics, and prognosis: a prospective cohort of breast cancer patients. Breast Cancer Res, 9, R6.

[5]. Ghosh J, Gupta S, Desai S, Shet T, Radhakrishnan S, Suryavanshi P et al. Estrogen, progesterone and HER2 receptor expression in breast tumors of patients, and their usage of HER2-targeted therapy, in a tertiary care centre in India. Indian J Cancer 2011;48:391-6

[6]. Ambroise M, Ghosh M, Mallikarjuna VS, Kurian A. Immunohistochemical Profile of Breast Cancer Patients at a Tertiary Care Hospital in South India Immunohistochemical Profile of Breast Cancer Patients at a Tertiary Care Hospital in South India. Asian Pacific J Cancer Prev 2011; 12:625-629

[7]. El Saghir NS, Seoud M, Khalil MK, et al. Effects of young age at presentation on survival in breast cancer. BMC Cancer. 2006;6:194.

[8]. Suvarchala SB, Nageshwararao R. Carcinoma Breast-Histopathological and hormone receptors correlation. J Biosci Tech 2011:2; 340-48.

[9]. Pakseresht S, Ingle G K, Bahadur A K, Ramteke V K, Singh M M, Garg S, Agarwal P N. Risk factors with breast cancer among women in Delhi. Indian J Cancer 2009; 46:132-8. 
[10]. Rhodes A, Jasani B, Balaton AJ, Barnes DM, Miller KD. Frequency of oestrogen and progesterone receptor positivity by immunohistochemical analysis in 7016 breast carcinomas: correlation with patient age, assay sensitivity, threshold value, and mammographic screening. J Clin Pathol. 2000; 53: 688-96.

[11]. Vinh-Hung V, Verschraegen C, Promish DI, et al. Ratios of involved nodes in early breast cancer. Breast Cancer Res. 2004;6:6808.

[12]. Veronesi U, Paganelli G, Viale G, et al. Sentinel Lymph Node Biopsy and Axillary Dissection in Breast Cancer: Results in a Large Series. J Natl Cancer Inst. 1999;91:368-73.

[13]. Jatoi I, Hilsenbeck SG, Clark GM, Osborne CK. Significance of Axillary Lymph Node Metastasis in Primary Breast Cancer. J Clin Oncol. 1999;17:2334-40.

[14]. Bland KI, Menck HR, Scott-Conner CE, Morrow M, Winchester DJ, Winchester DP. The national cancer data base 10-year survey of breast carcinoma treatment at hospitals in the United States. Cancer.1998;83:1262-73.

[15]. Carter CL, Allen C, Henson DE. Relation of tumor size, lymph node status, and survival in 24,740 breast cancer cases. Cancer. 1989;63:181-7. 